Research Paper

\title{
The Efficacy of Adjuvant Chemotherapy for Locally Advanced Upper Tract Urothelial Cell Carcinoma
}

\author{
Taek Sang Kim ${ }^{\bowtie}$, Jeong Hyun Oh, Hyun Yul Rhew \\ Department of Urology, College of Medicine, Kosin University Gospel Hospital, Busan, Korea. \\ $\triangle$ Corresponding author: Taek Sang Kim, address: Department of Urology, Kosin University Gospel Hospital, 34 Am Nam Dong, Seo GU, \\ Busan, Korea. E-mail: threeb74@naver.com Tel: +82-51-990-6279 Fax: +82-51-990-3994. \\ () Ivyspring International Publisher. This is an open-access article distributed under the terms of the Creative Commons License (http://creativecommons.org/ \\ licenses/by-nc-nd/3.0/). Reproduction is permitted for personal, noncommercial use, provided that the article is in whole, unmodified, and properly cited.
}

Received: 2013.08.02; Accepted: 2013.10.01; Published: 2013.10.05

\begin{abstract}
Objective: To evaluate the efficacy of adjuvant cisplatin-based chemotherapy for locally advanced upper tract urothelial cell carcinoma (UTUC) following radical nephroureterectomy with bladder cuff resection (RNU) in terms of survival and recurrence.

Materials and methods: Between January 2000 and January 2013, among 145 patients with upper tract urothelial cell carcinoma, a total of 65 patients with locally advanced UTUC (a diagnosis of $\mathrm{pT} 3$ or $\mathrm{pT} 4$ or $\mathrm{PTI}-2 \mathrm{NI}-3$ ) underwent RNU. Of these 65 patients, 36 patients received at least three cycles of adjuvant gemcitabine plus cisplatin chemotherapy and the remaining 29 patient did not receive adjuvant chemotherapy. Clinical characteristics, bladder recurrence, distant metastasis, and cancer-specific survival were retrospectively reviewed.

Results: The mean age of the 65 patients was 60.4 (range, 37-87) years and the median follow-up period was 34 (range, 12-114) months. Patent demographics were not statistically different between the two groups. During the follow-up period, 14 patients (21.5\%) experienced distant metastasis; $8(8 / 36,22.2 \%)$ patients who had undergone adjuvant chemotherapy and 6 (6/29, $20.7 \%$ ) patients who did not. Bladder recurrence was noted in 17 patients $(26.2 \%), 5(5 / 36,13.9 \%)$ of whom received adjuvant chemotherapy while the remaining $12(12 / 29,41.4 \%)$ did not. Kaplan-Meire and multivariate analysis showed that the incidence of bladder recurrence was significantly higher in patients who did not undergo adjuvant chemotherapy, and cancer specific survival was not significantly associated with adjuvant chemotherapy.

Conclusions: Adjuvant chemotherapy for locally advanced UTUC can prevent bladder recurrence, but has a minimal effect on cancer-specific survival.
\end{abstract}

Key words: urothelial cell carcinoma, chemotherapy, recurrence, survival.

\section{INTRODUCTION}

Upper tract urothelial carcinoma (UTUC) is a rare disease that accounts for approximately $5 \%$ of all urothelial malignancies, and radical nephroureterectomy with bladder cuff excision (RNU) is the standard treatment of UTUC ${ }^{1}$. The pathological features and clinical behavior of UTUC are very similar to those of bladder urothelial carcinoma, thus urologists consider them to be nearly the same disease. Tumor stage, nuclear grade, lymphovascular invasion, and node metastasis are valuable prognostic factors of UTUC. On the other hand, there are some controversies regard- ing the prognostic value of tumor location, tumor size, and adjuvant chemotherapy ${ }^{2-9}$. Studies have demonstrated the value of neoadjuvant chemotherapy for locally advanced bladder carcinoma ${ }^{10-12}$, and some series have reported a survival benefit of chemotherapy in metastatic UTUC ${ }^{13}$. Locally advanced non-metastatic UTUC is associated with poor survival, and there is no standardized therapy conferring a survival benefit after RNU. The only prospective randomized clinical trial - the POUT (peri-operative chemotherapy versus surveillance in upper tract 
urothelial cancer) trial just started in April 2012 in United Kingdom ${ }^{14}$. Some urologists administer adjuvant chemotherapy after RNU although there is a lack of data supporting its efficacy of in UTUC. Therefore, we assessed the significance of adjuvant chemotherapy in UTUC after RNU focusing on survival.

\section{MATERIAL AND METHODS}

The study flow chart is described in Fig. 1. After obtaining institutional review board approval, we retrospectively reviewed the medical records of 145 patients diagnosed with UTUC between January 2000 and January 2013. Among them, a total of 65 patients had non-metastatic, locally advanced upper tract urothelial cell carcinoma (pT3 or pT4 or pT1-2N1-3) after undergoing RNU. We performed radical nephroureterectomy with bladder cuff resection by laparoscopic and open approach. Hilar lymph node was routinely removed in all our procedures. In cases of presence of hyperplastic lymph nodes, beside the great vessel or hilum we tried to resect grossly visible or palpable lymph node as meticulously as possible. After discharge, patients re-visited out-patient clinic, then we explained the potential benefit (survival gain) and disadvantage of adjuvant chemotherapy (uncertainty of survival benefit, chemo-toxicities, medical co-morbidity, financial problem) to these patients. After gathering chemotherapy candidates, we checked creatinine clearance of these patients between 2 and 4 weeks after operation for making optimal dose of cisplatin. The first cycle of chemotherapy was performed within 4 weeks. A total of 36 patients received at least three cycles of adjuvant gemcitabine plus cisplatin chemotherapy while remaining 29 patients did not go through chemotherapy due to uncertainty of chemo-toxicities (13 patients),

Fig I. Study flow diagram. survival benefit (7 patients), medical co-morbidities ( 5 patients) and financial issues (4 patients). The chemotherapy patients were administered $1000 \mathrm{mg} / \mathrm{m}^{2}$ gemcitabine on day 1,8 , and 15 and $70 \mathrm{mg} / \mathrm{m}^{2}$ cisplatin on day 2 for the GC regimen. The dose of cisplatin was reduced from 50 to $70 \%$ of normal dose when estimated glomerular filtration rate was under $70 \mathrm{ml} /$ minutes. After RNU or adjuvant chemotherapy, the patients received follow-up of routine hematology and biochemistry, cystoscopy, urinary cytology, simple chest $x$-ray every three months for the first two years, every six months in the next two years, and annually thereafter. Abdominopelvic CT scan was performed every six months in the first two years then annually afterward. We retrospectively reviewed medical records for clinical data and radiologic findings. Pathologic stage and nuclear grade were analyzed according to the AJCC (American Joint Committee on Cancer) and 1998 WHO/International Society of Urologic Pathology consensus classification ${ }^{15}$.
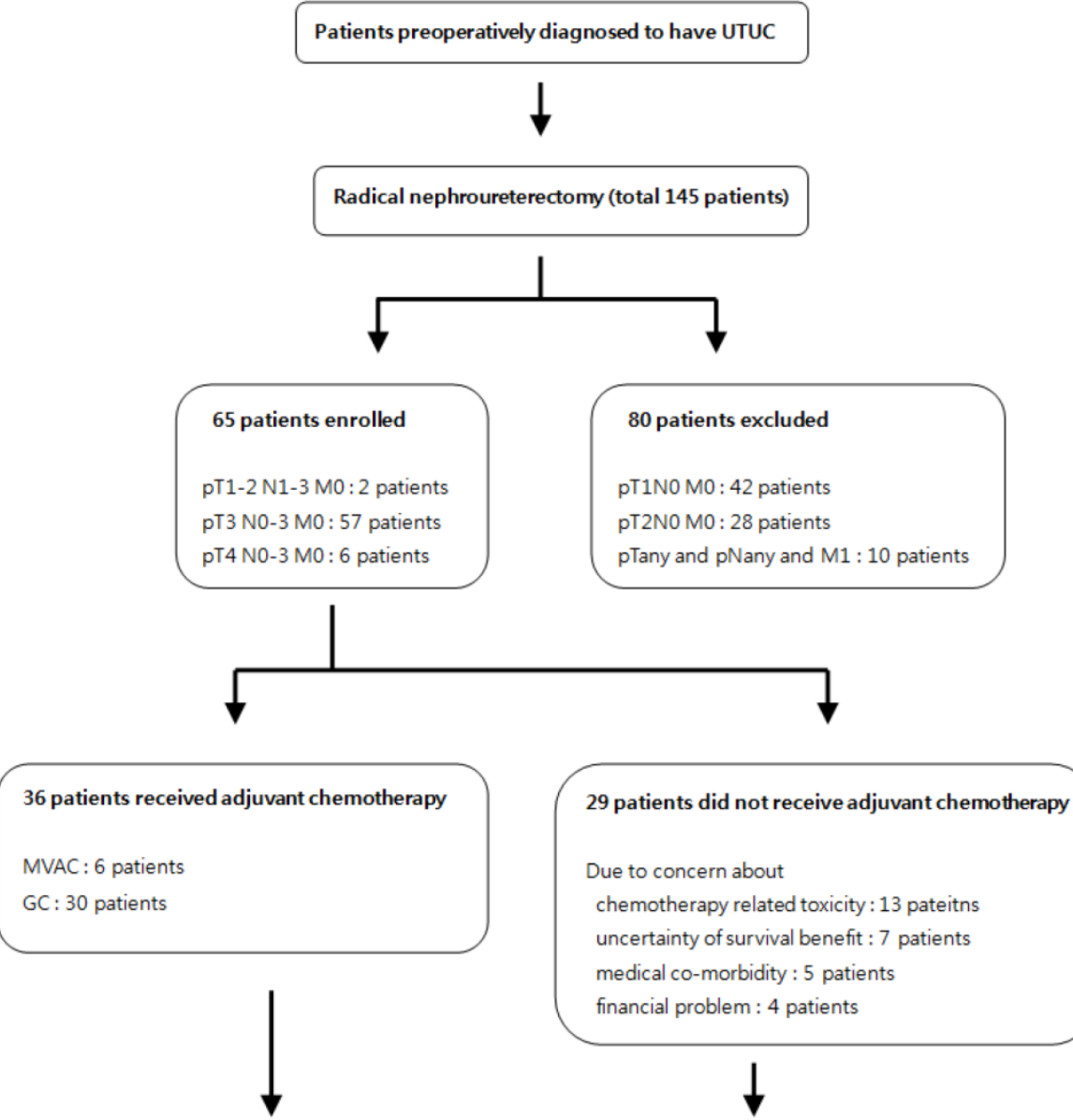
The characteristics of the chemotherapy group and non-chemotherapy group were analyzed using Fisher's exact test. Bladder recurrence free survival and cancer-specific survival were assessed using the Kaplan-Meier method and compared between the two groups using the log-rank test. The prognostic significance of clinical variables was evaluated by the Cox proportional hazards model, and a p-value $<0.05$ was considered statistically significant for all analyses.

\section{RESULTS}

\section{Patient characteristics}

The mean age of the 65 patients was 60.4 (range, 37-87) years and the median follow-up period was 34 (range, 12-114) months. Perioperative clinical data were not statistically different between the two groups, except bladder recurrence. Detailed characteristics of each patient group are summarized in Table 1.

\section{Recurrence and survival}

During follow-up period, 14 patients $(21.5 \%)$ experienced distant metastasis, $8(8 / 36,22.2 \%)$ of whom underwent adjuvant chemotherapy while the remaining $6(6 / 29,20.7 \%)$ patients did not. Bladder recurrence was noted in 17 patients $(26.2 \%)$ at a median of 8 months (range, 3-30), $5(5 / 36,13.9 \%)$ in the adjuvant chemotherapy group and $12(12 / 29,41.4 \%)$ in the group that did not receive chemotherapy. On multivariate cox proportional hazard model analysis, bladder recurrence was significantly higher in patients who did not undergo adjuvant chemotherapy $(p=0.001, H R=14.862)$, and cancer-specific survival was significantly associated with lymph node involvement $(p<0.001, H R=42.78)$. Cancer-specific survival was not associated with adjuvant chemotherapy (Tables 2). In Kaplan-Meier analysis, the bladder recurrence free survival was significantly higher in adjuvant chemotherapy group than non-chemotherapy group (log-rank test, $p=0.026)$. However, there was no significant difference in the cancer-specific survival (log-rank test, $\mathrm{p}=0.47$ ) (Figure 2).

Table I. The patients' characteristics.

\begin{tabular}{|c|c|c|c|c|}
\hline & $\begin{array}{l}\text { Chemo- } \\
\text { therapy } \\
(n=36)\end{array}$ & $\begin{array}{l}\text { No chem- } \\
\text { otherapy } \\
(n=29)\end{array}$ & $\begin{array}{l}\text { Total } \\
(\mathrm{n}=65)\end{array}$ & p-value \\
\hline Age (year) & & & & 0.273 \\
\hline$<60$ & 22 & 12 & 34 & \\
\hline$\geq 60$ & 14 & 17 & 31 & \\
\hline Gender & & & & 0.820 \\
\hline male & 19 & 21 & 40 & \\
\hline female & 17 & 8 & 25 & \\
\hline Tumor location & & & & 0.914 \\
\hline renal pelvis & 26 & 20 & 46 & \\
\hline ureter & 10 & 9 & 19 & \\
\hline Pathological tumor stage & & & & 0.125 \\
\hline pT1-2N1-3 & 2 & 0 & 2 & \\
\hline pT3N0-3 & 30 & 27 & 57 & \\
\hline pT4N0-3 & 4 & 2 & 6 & \\
\hline Nuclear grade (WHO) & & & & 0.388 \\
\hline grade $1 / 2$ & 4 & 6 & 10 & \\
\hline grade 3 & 32 & 23 & 55 & \\
\hline $\begin{array}{l}\text { Lymphovascular inva- } \\
\text { sion }\end{array}$ & & & & 0.266 \\
\hline$(-)$ & 22 & 16 & 38 & \\
\hline$(+)$ & 24 & 13 & 27 & \\
\hline Lymph node & & & & 0.458 \\
\hline$(-)$ & 28 & 26 & 54 & \\
\hline$(+)$ & 8 & 3 & 11 & \\
\hline Urine cytology & & & & 0.553 \\
\hline$(-)$ & 16 & 12 & 28 & \\
\hline$(+)$ & 20 & 17 & 37 & \\
\hline Bladder recurrence & & & & 0.001 \\
\hline$(-)$ & 31 & 17 & 48 & \\
\hline$(+)$ & 5 & 12 & 17 & \\
\hline $\begin{array}{l}\text { Distant metastasis dur- } \\
\text { ing follow-up }\end{array}$ & & & & 0.590 \\
\hline$(-)$ & 28 & 23 & 51 & \\
\hline$(+)$ & 8 & 6 & 14 & \\
\hline
\end{tabular}

Table 2. Multivariate analysis for predicting bladder recurrence and cancer-specific free survival.

\begin{tabular}{|c|c|c|c|c|c|c|}
\hline & \multicolumn{3}{|c|}{ Bladder recurrence } & \multicolumn{3}{|c|}{ Cancer-specific free survival } \\
\hline & HR & $95 \% \mathrm{CI}$ & p-value & HR & $95 \% \mathrm{CI}$ & p-value \\
\hline Age & 1.22 & $0.39-3.72$ & 0.631 & 1.50 & $0.75-3.53$ & 0.221 \\
\hline Gender & 0.23 & $0.03-1.84$ & 0.149 & 0.57 & $0.21-1.64$ & 0.171 \\
\hline Tumor location & 0.78 & $0.32-2.85$ & 0.482 & 1.62 & $0.54-6.21$ & 0.438 \\
\hline Nuclear grade & 1.84 & $0.46-7.35$ & 0.359 & 3.85 & $0.70-18.3$ & 0.094 \\
\hline LNI & 1.23 & $0.41-3.86$ & 0.761 & 42.78 & $8.7-210.36$ & 0.000 \\
\hline LVI & 0.82 & $0.16-6.10$ & 0.635 & 1.86 & $0.57-5.40$ & 0.337 \\
\hline Urine cytology & 1.78 & $0.43-7.26$ & 0.325 & 1.33 & $0.81-2.36$ & 0.110 \\
\hline ACT & 14.862 & $3.20-68.99$ & 0.001 & 1.94 & $0.55-5.77$ & 0.210 \\
\hline
\end{tabular}

HR : hazard ratio, CI : confidence interval, LNI : lymph node involvement, LVI : lymphovascular invasion, ACT : adjuvant chemotherapy. 


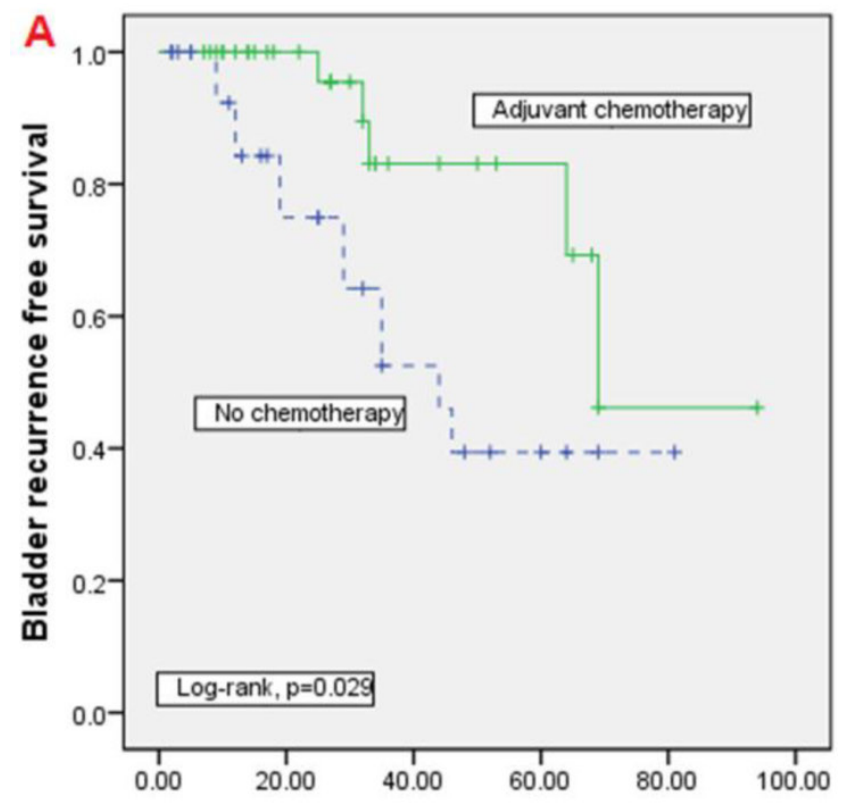

Time after surgery (months)

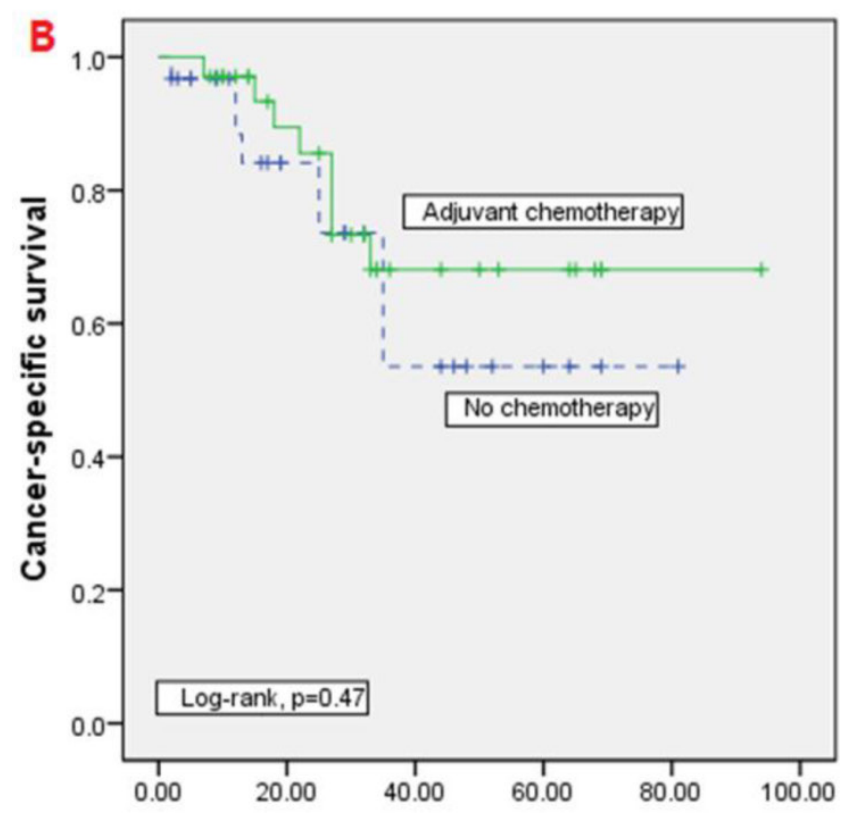

Time after surgery (months)

Fig 2. Kaplan-Meier analyses of bladder recurrence free survival and cancer-specific survival. In Kaplan-Meier analysis, bladder recurrence free survival was significantly higher in adjuvant chemotherapy group than non-chemotherapy group $(A$; log-rank test, $p=0.026)$. However, there was no significant difference in the cancer-specific survival (B; log-rank test, $\mathrm{p}=0.47)$.

\section{DISCUSSION}

Advanced UTUC is difficult to treat because of the poor survival rate and high rate of recurrence. The five-year survival rate of low stage (pT1) UTUC is known to be greater than $90 \%$. However, pT3 stage UTUC has a poor five-year survival rate of approximately $50 \%$, and this rate decreases to $5 \%$ in pT4 stage disease $^{2}$. This discrepancy in survival rate between low and high stage UTUC have led urologic oncologist to recommend further treatment.

The survival benefit of neoadjuvant chemotherapy before radical cystectomy in patients with invasive bladder carcinoma has been demonstrated ${ }^{10-12}$; however, the efficacy of adjuvant chemotherapy after radical cystectomy remains controversial ${ }^{9}$. As is the case for bladder carcinoma, there are limited data on the efficacy of adjuvant chemotherapy for UTUC. Until the results of POUT trials or other randomized prospective clinical trials are to be revealed, some will give adjuvant chemotherapy without any reliable data while others will not due to an uncertainty of benefit $^{14}$. Further complicating the issue, uro-oncologists may hesitate to administer chemotherapeutic agents after RNU based on concern over nephrotoxicity to the solitary kidney.

Kwak et al. suggested that adjuvant chemotherapy for high-risk UTUC (including stage pT2) can provide a therapeutic benefit ${ }^{16}$; however, Lee et al. reported that there was no significant difference in cancer-specific survival or recurrence-free survival between an adjuvant chemotherapy group and non-chemotherapy group ${ }^{17}$. According to the largest study to date conducted by Hellenthal, adjuvant chemotherapy has no survival benefit for high-risk UTUC $^{18}$. In our study, 19 patients died during the follow-up period due to disease progression; however, there was no significant difference in survival between the adjuvant chemotherapy and non-chemotherapy groups. Hisataki et al. reported that most instances of recurrence developed within two years after RNU, and the majority of bladder recurrence cases (19 of 22 bladder recurrent patients) in UTUC were non-muscle invasive bladder cancers ${ }^{19}$. Our study showed similar results. A total of 17 patients experienced bladder recurrence at a median of 8 months. However, all bladder recurrences were non-muscle invasive bladder cancers and could be treated by transurethral resection with intravesical BCG or mitomycin C instillation.

Cho et al. reported that baseline creatinine clearance decreased below $60 \mathrm{ml} / \mathrm{min}$ in $83.3 \%$ of patients after nephroureterectomy. However, the majority of patients with a solitary kidney underwent cisplatin-based chemotherapy without serious adverse events ${ }^{20}$. We also believe that nephrotoxicity to the single renal unit is not a major concern in adjuvant chemotherapy. In our analysis, known prognostic factors such as lymphovascular invasion, tumor location (ureter or renal pelvis), and nuclear grade were not significantly associated with survival. These results may be due to the fact that we included only patient with stage pT3 or pT4 disease. If we had in- 
cluded cases of pT1 or pT2 disease, these prognostic factors may have been significant. Some investigators reported that tumor location is an independent risk factor for bladder recurrence ${ }^{21,22}$. On the other hand, others suggested that multifocality and concomitant CIS are the significant risk factors ${ }^{23}$. In our study, these known risk factors for bladder recurrence were not significant; adjuvant chemotherapy was the only significant factor for bladder recurrence. The studies mentioned above included all stages of UTUC, and the fact that we included only cases with high stage disease is likely the reason underlying the lack of statistical significance observed between commonly accepted prognostic factors and recurrence or survival. Soga et al. reported that adjuvant M-VAC chemotherapy reduced bladder recurrence in stage pT2 or higher UPTC ${ }^{24}$. That study was performed over 20 years; patients received adjuvant chemotherapy during the first 10 years of the study period while they did not during the latter 10 years. The fact that the chemotherapy and non-chemotherapy groups were not enrolled during the same period indicates the potential for selection bias.

According to our study, cisplatin-based adjuvant chemotherapy for UTUC has no influence on cancer-specific survival. However, adjuvant chemotherapy can reduce bladder recurrence, thereby preventing transurethral resection of recurrent bladder tumors and intravesical therapy. Nevertheless, bladder recurrence was not associated with cancer-specific survival because these patients were cured with transurethral resection of the recurrent bladder tumor with or without intravesical therapy. If patients with advanced UTUC do not receive adjuvant chemotherapy, there is a higher possibility of bladder recurrence. However, adjuvant chemotherapy and bladder recurrence did not have a significant effect on cancer-specific survival in this study. Uro-oncologists should consider the toxicities of chemotherapy and patient compliance and financial status when determining whether chemotherapy is appropriate.

\section{CONCLUSION}

Due to similarities with the pathological features of bladder carcinoma, adjuvant chemotherapy for locally advanced UTUC is often suggested. However, our findings suggest that adjuvant chemotherapy for locally advanced UTUC can prevent bladder recurrence but has a minimal effect on cancer-specific survival. Prospective randomized clinical trials are necessary to verify the effect of adjuvant chemotherapy on locally advanced UTUC.

\section{Competing Interests}

The authors have declared that no competing interest exists.

\section{References}

1. Tawfiek ER, Bagley DH. Upper-tract transitional cell carcinoma. Urology. 1997; 50: 321-9.

2. Hall MC, Womack S, Sagalowsky AI, et al. Prognostic factors, recurrence, and survival in transitional cell carcinoma of the upper urinary tract: a 30-year experience in 252 patients. Urology. 1998; 52: 594-601.

3. Cho DS, Hong SY, Kim YK, et al. Prognostic factors in transitional cell carcinoma of the upper urinary tract after radical nephroureterectomy. Korean J Urol. 2011; 52: 310-6.

4. Park S, Hong B, Kim CS, et al. The impact of tumor location on prognosis of transitional cell carcinoma of the upper urinary tract. J Urol. 2004; 171: 621-5.

5. Kikuchi E, Margulis V, Karakiewicz PI, et al. Lymphovascular invasion predicts clinical outcomes in patients with node-negative upper tract urothelial carcinoma. J Clin Oncol. 2009; 27: 612-8.

6. Favaretto RL, Shariat SF, Chade DC, et al. The effect of tumor location on prognosis in patients treated with radical nephroureterectomy at Memorial Sloan-Kettering Cancer Center. Eur Urol. 2010; 58: 574-80.

7. Novara G, Matsumoto K, Kassouf W, et al. Prognostic role of lymphovascular invasion in patients with urothelial carcinoma of the upper urinary tract: an international validation study. Eur Urol. 2010; 57: 1064-71.

8. Yafi FA, Novara G, Shariat SF, et al. Impact of tumour location versus multifocality in patients with upper tract urothelial carcinoma treated with nephroureterectomy and bladder cuff excision: a homogeneous series without perioperative chemotherapy. BJU Int. 2012; 110: E7-13.

9. Advanced Bladder Cancer (ABC) Meta-analysis Collaboration. Adjuvant chemotherapy in invasive bladder cancer: a systematic review and meta-analysis of individual patient data Advanced Bladder Cancer (ABC) Meta-analysis Collaboration. Eur Urol. 2005; 48: 189-201.

10. Grossman HB, Natale RB, Tangen CM, et al. Neoadjuvant chemotherapy plus cystectomy compared with cystectomy alone for locally advanced bladder cancer. N Engl J Med. 2003; 349: 859-66.

11. Advanced Bladder Cancer (ABC) Meta-analysis Collaboration. Neoadjuvant chemotherapy in invasive bladder cancer: update of a systematic review and meta-analysis of individual patient data advanced bladder cancer (ABC) meta-analysis collaboration. Eur Urol. 2005; 48: 202-6.

12. Winquist E, Kirchner TS, Segal R, et al. Neoadjuvant chemotherapy for transitional cell carcinoma of the bladder: a systematic review and meta-analysis. J Urol. 2004; 171: 561-9.

13. Tannock I, Gospodarowicz M, Connolly J, et al. M-VAC (methotrexate, vinblastine, doxorubicin and cisplatin) chemotherapy for transitional cell carcinoma: the Princess Margaret Hospital experience. J Urol. 1989; 142: 289-92.

14. Birtle AJ, Lewis R, Johnson M, et al. Time to define an international standard of postoperative care for resected upper urinary tract transitional cell carcinoma (TCC) - opening of the peri-operative chemotherapy versus surveillance in upper tract urothelial cancer (POUT) Trial. BJU Int. 2012; 110: 919-21.

15. Epstein JI, Amin MB, Reuter VR, et al. The World Health Organization/International Society of Urological Pathology consensus classification of urothelial (transitional cell) neoplasms of the urinary bladder. Bladder Consensus Conference Committee. Am J Surg Pathol. 1998; 22: 1435-48.

16. Kwak C, Lee SE, Jeong IG, et al. Adjuvant systemic chemotherapy in the treatment of patients with invasive transitional cell carcinoma of the upper urinary tract. Urology. 2006; 68: 53-7.

17. Lee SE, Byun SS, Park YH, et al. Adjuvant chemotherapy in the management of pT3NOM0 transitional cell carcinoma of the upper urinary tract. Urol Int. 2006; 77: 22-6.

18. Hellenthal NJ, Shariat SF, Margulis V, et al. Adjuvant chemotherapy for high risk upper tract urothelial carcinoma: results from the Upper Tract Urothelial Carcinoma Collaboration. J Urol. 2009; 182: 900-6.

19. Hisataki T, Miyao N, Masumori N, et al. Risk factors for the development of bladder cancer after upper tract urothelial cancer. Urology. 2000; 55: 663-7.

20. Cho KS, Joung JY, Seo HK, et al. Renal safety and efficacy of cisplatin-based chemotherapy in patients with a solitary kidney after nephroureterectomy for urothelial carcinoma of the upper urinary tract. Cancer Chemother Pharmacol. 2011; 67: 769-74

21. Zigeuner RE, Hutterer G, Chromecki T, et al. Bladder tumour development after urothelial carcinoma of the upper urinary tract is related to primary tumour location. BJU Int. 2006; 98: 1181-6.

22. Cosentino M, Palou J, Gaya JM, et al. Upper urinary tract urothelial cell carcinoma: location as a predictive factor for concomitant bladder carcinoma. World J Urol. 2013; 31: 141-5.

23. Xylinas E, Colin P, Audenet F, et al. Intravesical recurrence after radical nephroureterectomy for upper tract urothelial carcinomas: predictors and impact on subsequent oncological outcomes from a national multicenter study. World J Urol. 2013; 31: 61-8.

24. Soga N, Arima K, Sugimura Y. Adjuvant methotrexate, vinblastine, adriamycin, and cisplatin chemotherapy has potential to prevent recurrence of bladder tumors after surgical removal of upper urinary tract transitional cell carcinoma. Int J Urol. 2008; 15: 800-3. 